\section{Verhaltenstherapie}

Adam-Schwebe, S. 45

Alder, J. 27

Alpers, G.W. 6,11

Archonti, C. 2, 27

Arck, P. 20

Aulhorn, I. 27

Bach, D. 28

Bachmann, M. 20

Bailer, J. 47

Bailer, U. 17

Balke, B. 44

Ballauff, A. 18

Baltruschat, N. 28

Barbir, A. 14

Barr Taylor, C. 11

Batra, A. 5

Bauer, A. 4

Bauernschmitt, K. 28

Baumeister, H. 10

Becker, E. 29, 43

Beintner, I. 30, 40

Beisel, S. 29

Bengel, J. 10

Berger, S. 42

Berking, M. 29

Biedert, E. 19

Bieniakiewicz, I. 14

Bischoff, C. 45

Bitzer, E. 44

Bitzer, J. 27

Blättler, N. 35

Bleichhardt, G. 30

Boisten, C.J.M. 32

Bosy-Westphal, A. 18

Brabant, G. 18

Braus, D. 38

Brinkmeyer, J. 39

Broutschek, B. 37

Brunk, J. 14

Bruns, T. 29

Bürger, I. 30, 40

Castellanos, H. 34

Cebulla, M. 16, 29, 34, 40

Classen, C. 11

Coles, J. 31

\title{
Autorenverzeichnis - Author Index
}

Verhaltenstherapie 2003;13(suppl1):49-51

Crössmann, A. 31

Cuntz, U. 16

Czikkely, M. 45

D'Amelio, R. 2, 27, 32

Dahme, B. 34, 41, 46

Dannigkeit, N. 31

de Zwaan, M. 17

Degener, S. 32

Delb, W. 2, 32

Demmel, R. 27, 43

Dev, P. 11

Dignass, A. 20

Dilger, S. 32

Dohrenbusch, R. 33

Dörning, H. 44

Drinkmann, A. 6

Eberl, A. 33

Eger, K. 46

Ehlert, U. 14, 35, 35, 42

Eickelmann, S. 25

Ellwart, T. 43

Enck, P. 21, 37

Erdmann, G. 28

Exner, C. 18

Falkai, P. 2, 27

Fallgatter, A.J. 7, 36

Fanke, J. 33

Fehm, H.L. 18

Fehm, L. 33

Fehm-Wolfsdorf, G. 8

Ferstl, R. 20

Fichter, M.M. 10, 11, 16, 16, 18, 18, 29, $34,35,39,40$

Fiegenbaum, W. 26

Fikentscher, E. 44

Frank, M. 26

Franz, U. 34

Freudenberg, G. 34

Friederich, H.M. 5

Frodl, T. 38

Frommann, I. 38

Frommann, N. 39

Fruehauf, E. 16

Fumi, M. 16
Gaab, J. 35, 35, 42

Gatterer, G. 14

Gehring, U. 6

Geissner, E. 28, 34

Gembruch, U. 14

Gemende, I. 37

Gerber, W.-D. 4, 36, 40, 41

Gerber-von Müller, G. 36

Gerhards, F. 1, 2

Gerlach, A.L. 7

Germann, N. 14

Goebel, G. 1

Göhringer, T. 8

Goldmann, D. 20

Gottschalk, A. 35

Graul, J. 1

Grosse Holforth, M. 29

Güner, P. 35

Haak, T. 36

Haas, V. 18

Habel, U. 38, 39

Haerkötter, C. 4

Hahlweg, K. 26

Hajak, G. 22, 22, 23

Hall, G. 37

Hallqvist, J. 43

Hamm, A.O. 7

Hampel, P. 4

Hanewinkel, R. 36

Härter, M. 10

Hartmann, M. 41

Hasselmeyer, A. 1

Hauer, M. 18

Hauerwas, D. 14

Hauser, C. 13

Haustein, K.-O. 5

Hautzinger, M. 40

Hebebrand, J. 18

Hebisch, G. 13

Hegar, K. 8

Heinrichs, M. 13, 13

Heinrichs, N. 26

Helbig, S. 33

Heldwein, W. 16

Henn, F.A. 38

Hermanns, N. 10, 36 
Herpertz, S. 18

Herpertz-Dahlmann, B. 18

Herrmann, M.J. 7, 36

Heuser, J. 1, 39

Hilbert, A. 20

Hiller, W. 1, 4, 10, 21, 30

Hinninghofen, H. 21

Hoffmeister, S.

Holtkamp, C. 18

Hoyer, J. 39

Ihle, D. 11

Isensee, B. 36

Jacob, C. 7, 36

Jacobi, C. 29

Jäger, B. 37

Jäkel, K. 37

Jewell, K. 31

Jud, A. 42

Jung, J. 27

Karl, A. 47

Kaspers, F.A. 33

Kathmann, N. 38

Kaufmann, B. 38

Kellermann, S. 37

Kellermann, T. 39

Kellner, A. 38

Kemmesies, U.E. 6

Kircher, T. 38

Kirschbaum, C. 42

Klapp, B.F. 20

Klein, H. 18

Klein, M. 28, 38, 39

Klein, T. 27

Klingenspor, M. 18

Klinger, R. 1, 24, 34, 38, 41

Klonis, T. 35

Klose, M. 39

Klosterhalfen, S. 37

Kluth, W. 39

Koch, K. 39

Koch, T. 37

Koch, U. 39

Kohlböck, G. 34

König, D. 6

Konzag, T.A. 44

Koopman, C. 11

Korb, U. 4

Körkel, J. 6

Korn, H.-J. 40

Köster, G. 31

Kowalcek, I. 14
Kramer, B. 43

Krampe, H. 15

Kränzlin, N. 16

Krause, P. 22

Krebs, H. 31

Krenn, H. 18

Kriechbaum, M. 36

Kröner-Herwig, B. 2, 4, 29, 30, 40

Kropp, P. 36, 40, 41

Kruse, J. 8

Kubiak, T. 36

Küfner, H. 15

Kulfanek, M. 26

Kulzer, B. 10, 36

Küpper, B. 22

Lammers, C. 14

Lamprecht, F. 23, 37

Langewitz, W. 24

Langs, G. 12

Legenbauer, T. 41

Lehmann, C. 41

Lehnert, P. 16

Leibbrand, R. 21

Lempa, W. 23

Leplow, B. 12, 19, 33, 37, 43, 46, 46

Limbacher, K. 45

Lincoln, T. 26

Linden, M. 26

Lindenmeyer, J. 16

Lippke, S. 42

Ludolph, A.C. 46

Maercker, A. 47

Maes, M. 11

Malewski, P. 37

Mallwitz, J. 34

Manthey, S. 18

Matter, N. 41

Maxeiner, S. 26

Meisenzahl, E. 38

Meller, I. 34

Melzig, C.A. 7

Menzi, T. 35

Merten, F. 42

Middendorf, T. 12

Miltner, W. 32

Mohrad, E. 23, 24

Möller, W.-D. 40

Mönnikes, H. 20

Morris, L. 32, 42

Moschner, C. 26

Moser, G. 21

Mühlberger, A. 7, 12
Müller, B. 38

Müller, G. 34, 41

Müller, M. 38

Müller, M.J. 18

Müller, T. 45

Munsch, S. 19

Murphy, R. 19

Mussgay, L. 23, 24

Naab, S. 16

Nanke, A. 11

Nater, U.M. 42

Neubauer, G. 15

Neudecker, S. 43

Nickel, L. 23

Nicolai, J. 43

Niedermeier, T. 34

Noeker, M. 5

Nutzinger, D.O. 1, 12, 18, 18, 19, 19, 20

Oettingen, G. 41

Otto, B. 16

Pabst, B. 35

Patak, M. 2

Paul, T. 18, 19, 19, 20

Pauli, P. 7, 12, 31

Peter, G. 20

Peter, R. 43

Petermann, F. 4

Pfingsten, M. 25

Pilger, F. 11

Pittrow, D. 22

Plate, U. 12

Plinkert, P.K. 2, 32

Priemer, B. 31

Pudel, V. 32, 42

Quadflieg, N. 18, 34

Rabe, S. 47

Rauh, E. 11

Raum, E. 44

Reinecke, S. 5

Reinecker, H. 28

Remschmidt, H. 18

Renner, B. 45

Richter-Appelt, H. 5

Rief, W. 11, 11, 11, 35

Riemann, D. 22, 22

Rinck, M. 29, 43

Rist, F. 7, 27, 43, 47

Roberts, H. 11

Robra, B.-P. 44 
Rockahr, A. 43

Rodenbeck, A. 23

Rohleder, N. 42

Roth, W.T. 6

Rübler, D. 44

Rüddel, H. 23, 24, 26, 27, 41

Ruhmland, M. 30, 40

Ruhrmann, S. 38

Rustenbach, S. 1

Rüther, E. 23

Sack, M. 23

Sauer, H. 38

Schaaf, L. 8

Schächinger, H. 8, 24

Schlösser, R. 38

Schmidt, F. 23, 24

Schmidt, T. 44, 44

Schmieder, R. 26

Schmitt, A. 38

Schmitz, N. 8

Schneider, F. 38, 39

Schön, D. 5

Scholz, O.B. 33

Schramm, S. 41

Schröder, B. 26

Schroeter, K. 19

Schubert, K. 32

Schultze, H. 45

Schulz, H. 45

Schupp, H.T. 7

Schumacher, H.-L. 39

Schürmeyer, T. 35

Schwab, B. 37

Schwartz, F.W. 44

Schweiger, U. 18

Schwerdtfeger, F.-P. 1
Schwiersch, M. 28

Seelbach, H. 34

Sellmer, I. 20

Senf, W. 18

Sevignani, N. 34

Shah, N.J. 38, 39

Sieber, S. 14

Siegrist, J. 43

Siniatchkin, M. 41

Smesny, S. 38

Soost, S. 24

Sosnovsky, N. 34

Standl, E. 8

Stangier, U. 45

Stauber, T. 4

Stockhorst, U. 37

Stoyer, S. 35

Straube, T. 32

Streit, M. 39

Strube, D. 46

Telschow, A. 46

Tendolkar, I. 38

Thefeld, W. 8

Theorell, T. 43

Thienel, R. 38

Tschöp, M. 16

Tuschen-Caffier, B. 20, 31

Unterecker, S. 7, 36

Upatel, T. 7

v. Pein, A. 45

Vetter, P. 40

Voelker, W. 31

Vögele, C. 3, 41, 45, 47

von Leupoldt, A. 46 von Witzleben, I. 26

Vorhold, V. 47

Wagner, M. 38

Walter, A. 15

Walter, U. 44

Warnke, A. 18

Weidemann, F. 44

Weik, A. 12

Weike, A.I. 7

Weiss, T. 47

Weisz, N.

Wendt, A. 25

Wiedemann, G. 7,12

Wienbruch, C.

Wietek, B. 21

Wilhelm, F.H. 6

Wilhelm, H. 33

Winzelberg, A.J. 11

Wittchen, H.-U. 22

Witthöft, M. 47

Wolf, C. 44

Wolter, M. 45

Wölwer, W. 39

Worm, M. 24

Zachriat, C. 2, 4

Zapotoczky, H.G. 12

Ziegelmann, J.P. 42

Zierz, S. 43, 46

Zihl, J. 8

Zilles, K. 39

Zimmermann, R. 13

Zöllner, T. 47

Zschätsch, I. 5 